\title{
Pharmacokinetics and Bioavailability of a Fixed-Dose Combination of Ibuprofen and Paracetamol after Intravenous and Oral Administration
}

\author{
Hartley C. Atkinson ${ }^{1} \cdot$ Ioana Stanescu $^{1}$ - Chris Frampton ${ }^{2} \cdot$ Isam I. Salem $^{3}$ • \\ Charles P. H. Beasley ${ }^{1} \cdot$ Richard Robson $^{4}$
}

Published online: 3 September 2015

(c) The Author(s) 2015. This article is published with open access at Springerlink.com

\begin{abstract}
Background and Objectives Previously published studies have suggested the lack of a pharmacokinetic interaction between ibuprofen and paracetamol when they are delivered as a fixed-dose oral combination. The aim of this study was to determine the pharmacokinetic profile and safety of a fixed-dose intravenous (IV) combination, containing $3 \mathrm{mg} / \mathrm{mL}$ ibuprofen and $10 \mathrm{mg} / \mathrm{mL}$ paracetamol, in comparison with its individual components. The study also assessed the relative bioavailability of the same doses of the active ingredients when they were administered as an oral formulation.

Methods A single-dose, open-label, randomized, fiveperiod cross-over sequence pharmacokinetic study was undertaken in 30 healthy volunteers. Serial plasma samples were assayed for both paracetamol and ibuprofen concentrations, using validated liquid chromatography-tandem mass spectrometry methods. Pharmacokinetic parameters were computed using standard non-compartmental
\end{abstract}

This study was registered on the Australian New Zealand Clinical Trials Registry (trial ID: ACTRN12614000809639).

Richard Robson

richard@ccst.co.nz

Charles P. H. Beasley

charles.beasley@aftpharm.com

1 AFT Pharmaceuticals Ltd, PO Box 33203, Takapuna, Auckland 0740, New Zealand

2 University of Otago, PO Box 4345, Christchurch 8140, New Zealand

3 International Pharmaceutical Research Centre, 1 Queen Rania Street-Sport City Circle, Amman 11196, Jordan

4 Christchurch Clinical Studies Trust Ltd, PO Box 2856, Christchurch 8140, New Zealand analyses. Adverse events were also assessed. The ratios of the maximum measured plasma concentration $\left(C_{\max }\right)$, the area under the plasma concentration-time curve (AUC) from time zero to the time of the last measurable plasma concentration $\left(\mathrm{AUC}_{t}\right)$ and $\mathrm{AUC}$ from time zero to infinity $\left(\mathrm{AUC}_{\infty}\right)$ were analysed for bioequivalence as determined by $90 \%$ confidence intervals.

Results The pharmacokinetic parameters of ibuprofen and paracetamol were very similar for the combination and monotherapy IV preparations; the ratios of the $C_{\max }, \mathrm{AUC}_{t}$ and $\mathrm{AUC}_{\infty}$ values fell within the $80-125 \%$ acceptable bioequivalence range. Precise dose proportionality for both compounds was also determined for the half dose of the IV formulation in comparison with the full dose. The relative bioavailability of paracetamol $(93.78 \%)$ and ibuprofen $(96.45 \%)$ confirmed the pharmacokinetic equivalence of the oral and IV formulations of the fixed-dose combination. Conclusion Concomitant administration of $3 \mathrm{mg} / \mathrm{mL}$ ibuprofen and $10 \mathrm{mg} / \mathrm{mL}$ paracetamol in a fixed-dose IV combination does not alter the pharmacokinetic profiles of either drug. The IV and oral dose forms of such a combination are pharmacokinetically equivalent.

\section{Key Points}

Concomitant administration of $3 \mathrm{mg} / \mathrm{mL}$ ibuprofen and $10 \mathrm{mg} / \mathrm{mL}$ paracetamol in a fixed-dose intravenous combination does not alter the pharmacokinetic profile of either drug.

The intravenous and oral dose forms of such a combination are pharmacokinetically equivalent. 


\section{Introduction}

Ibuprofen and paracetamol are among the most commonly used analgesics and are widely available without a prescription [1]. Ibuprofen is a non-steroidal anti-inflammatory drug (NSAID), which non-selectively inhibits cyclooxygenase isozymes 1 and 2 (COX-1 and COX-2), resulting in inhibition of prostaglandins and related compounds at peripheral sites [2]. Contrastingly, the mode of action of paracetamol is not fully understood but is thought to relate to inhibition of either prostaglandin synthesis or cannabinoid receptors [3].

A fixed-dose combination of ibuprofen $300 \mathrm{mg}+$ paracetamol $1000 \mathrm{mg}$ (FDC 300/1000), when formulated as oral tablets (henceforth referred to as 'FDCoral'), has been shown to provide superior pain relief in comparison with its individual components [4]. An intravenous (IV) formulation of FDC 300/1000 (henceforth referred to as 'FDC-IV') has been developed to permit its administration to patients in whom the use of oral analgesics is limited by various patient factors, such as inability to swallow, the presence of postoperative nausea and vomiting, or reduced gastric motility.

Previous studies have demonstrated that oral ibuprofen and paracetamol are rapidly absorbed after oral administration and are not subject to significant first-pass metabolism [5-9], and that concomitant administration of both compounds does not result in a pharmacokinetic interaction [10-12]. In comparison with oral dosing, the same doses of ibuprofen and paracetamol administered in an IV formulation result in twofold and $70 \%$ increases in the maximum measured plasma concentration $\left(C_{\max }\right)$ values, respectively $[5,13]$. Furthermore, IV ibuprofen demonstrates great dose proportionality with respect to the area under the plasma concentration-time curve (AUC) and $C_{\max }[6,14]$.

The results of this study, which was conducted with aims to (1) describe the pharmacokinetic profile of the IV formulation of FDC-IV; (2) confirm the lack of a pharmacokinetic interaction between ibuprofen and paracetamol following IV administration; (3) determine whether dose proportionality is apparent from comparison of the full-dose combination of FDC-IV and the half-dose combination; and (4) establish the relative bioavailability of FDC-oral versus that of FDC-IV, are reported here.

\section{Methods}

\subsection{Trial Design}

This study was a phase I, single-centre, single-dose, openlabel, randomized, five-way cross-over trial in 30 healthy adult participants. After an initial screening period of up to 28 days, there were five study periods, separated by washout periods of at least $48 \mathrm{~h}$, and a subsequent final follow-up period of up to 7 days. The research was conducted in accordance with Good Clinical Practice (GCP), including the Declaration of Helsinki and all applicable regulatory requirements. The study protocol received approval by the Health and Disability Ethics Committee, Ministry of Health, New Zealand, and the trial was registered with the Australian New Zealand Clinical Trial Registry (trial ID: ACTRN12614000809639).

\subsection{Study Population}

Healthy volunteers of both genders, $18-50$ years of age with a body mass index (BMI) of $18.0-32.0 \mathrm{~kg} / \mathrm{m}^{2}$, were recruited from the Christchurch Clinical Studies Trust databases. Informed consent was obtained by the principal investigator from all individual participants included in the study prior to the screening visit. Screening involved a physical examination and recording of demographic data, vital signs, the medical history and concomitant medications. A blood sample was taken for haematology, biochemistry and serology screening, and a urine sample was collected for urinalysis, and illicit-drug and alcohol breath screening tests were performed. Suitable participants had to comply with all study inclusion and exclusion criteria (presented in Table 1).

\subsection{Treatment and Study Procedures}

Participants were randomized in a cross-over fashion to a study sequence of four IV doses and one oral dose, using a computer-generated list prior to commencement. For all doses, participants were confined to the study centre from the previous evening to approximately $12 \mathrm{~h}$ after study drug administration. The study drug was administered in the morning, following a 10-h fast, and a standard lunch and snacks were provided at 4 and $8 \mathrm{~h}$ after study drug administration, respectively.

Patients received each of the following five treatments in a randomized order:

- Treatment A (FDC-IV: $3 \mathrm{mg} / \mathrm{mL}$ ibuprofen $+10 \mathrm{mg} /$ $\mathrm{mL}$ paracetamol, $100 \mathrm{~mL} \mathrm{IV)}$

- Treatment B (10 mg/mL paracetamol, $100 \mathrm{~mL}$ IV)

- Treatment C (3 mg/mL ibuprofen, $100 \mathrm{~mL} \mathrm{IV)}$

- Treatment D (FDC-IV half dose: $1.5 \mathrm{mg} / \mathrm{mL}$ ibuprofen $+5 \mathrm{mg} / \mathrm{mL}$ paracetamol, $100 \mathrm{~mL} \mathrm{IV)}$

- Treatment E (FDC-oral: ibuprofen $150 \mathrm{mg}+$ paracetamol $500 \mathrm{mg}$ per tablet, 2 tablets)

Treatments A, B, C and D were manufactured by SM Farmaceutici SRL, Italy, and administered as a slow 
Table 1 Study inclusion/exclusion criteria

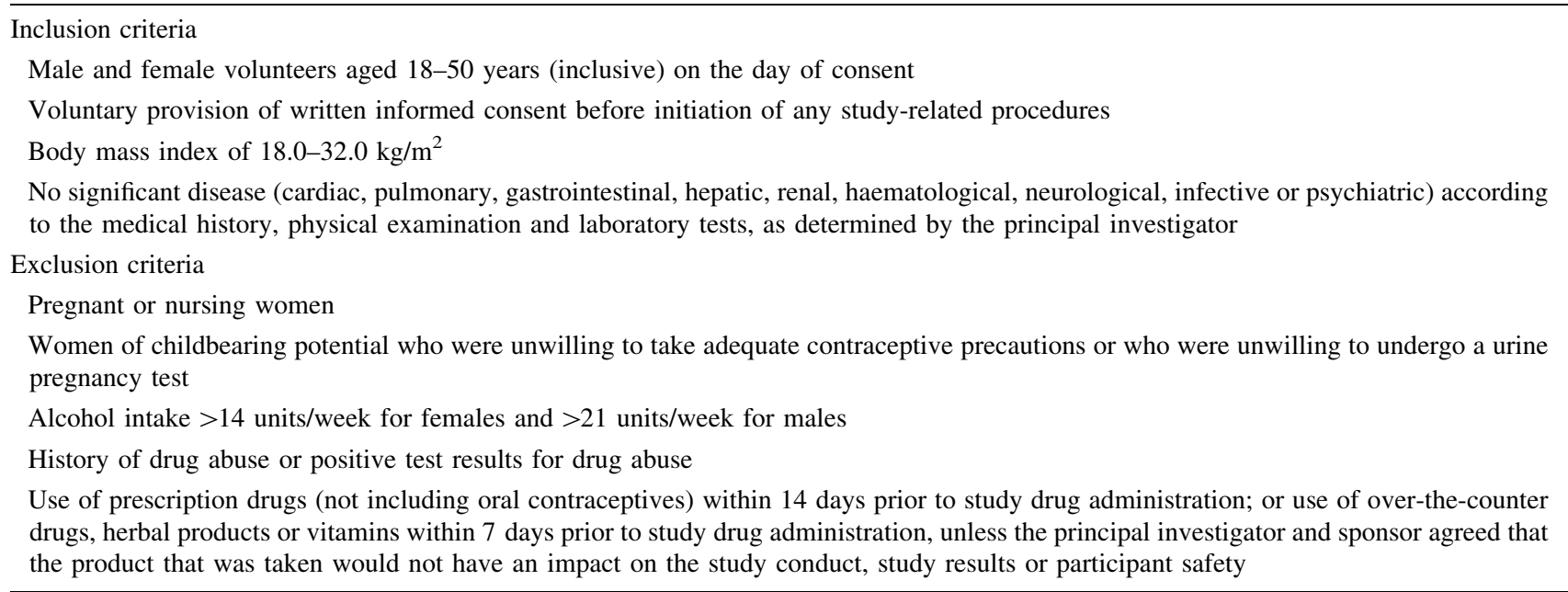

IV infusion over $15 \mathrm{~min}$ into an indwelling cannula. Treatment E was manufactured by Sigma Laboratories, India, and administered orally with $240 \mathrm{~mL}$ of water.

\subsection{Sampling Schedule}

Blood samples (approximately $5 \mathrm{~mL}$ ) for pharmacokinetic analysis were collected into lithium-heparin tubes. The blood sampling schedules were different for the IV and tablet formulations, as outlined below:

Sampling time points for the intravenous formulations Blood samples were drawn pre-dose, on completion of the 15-min IV infusion, at 5, 10, 15, 20, 30 and $45 \mathrm{~min}$, and at $1,1.25,1.5,2,3,4,6,8,10$ and $12 \mathrm{~h}$ postcompletion of the infusion.

Sampling time points for the tablet formulation Blood samples were drawn pre-dose, at 5, 10, 20, 30 and $45 \mathrm{~min}$, and at 1, 1.25, 1.5, 2, 3, 4, 6, 8, 10 and $12 \mathrm{~h}$ after study drug administration.

\subsection{Bioanalytical Methods}

All haematology, biochemistry and urinary analyses were conducted using standard methodologies within a single laboratory. Plasma concentrations of paracetamol and ibuprofen in human plasma (lithium-heparin) were determined using validated liquid chromatography-tandem mass spectrometry (LC-MS/MS) procedures.

Paracetamol plasma concentrations were analysed using API 3000 and Quattro premier mass spectrometry in multiple reaction monitoring (MRM) mode, using a turbo ion spray with positive ionization. The chromatographic separation of paracetamol employed a $\mathrm{C}_{18}$ column, using a mobile phase consisting of de-ionized water, formic acid and acetonitrile. The calibration curves were linear over the working range of $50-20,000 \mathrm{ng} / \mathrm{mL}$, with a regression coefficient $\left(R^{2}\right)$ of $\geq 0.99937$. The lower limit of quantification (LLOQ) was $50 \mathrm{ng} / \mathrm{mL}$ (precision $3.44 \%$, accuracy $93.69 \%)$.

Ibuprofen plasma concentrations were analysed using a Sciex API 3000 and API 4000 triple quadrupole mass spectrometer in MRM mode, using a turbo ion spray with negative ionization. Selective analysis of ibuprofen was achieved on a Symmetry $\mathrm{C}_{18}$ column by using a mobile phase consisting of ammonium formate, methanol and acetonitrile. The calibration curves were linear over the concentration range of $50-35,000 \mathrm{ng} / \mathrm{mL}$, with an $R^{2}$ of $\geq 0.9975$. The LLOQ was $50 \mathrm{ng} / \mathrm{mL}$ (precision $8.85 \%$, accuracy $104.25 \%$ ). These methods have also been described elsewhere [10].

\subsection{Pharmacokinetic Analysis}

The pharmacokinetic parameters of paracetamol and ibuprofen were calculated using Excel 2013. $C_{\max }$ and the time to reach $C_{\max }\left(T_{\max }\right)$ were determined directly from the raw data. The elimination rate constant $\left(k_{\mathrm{e}}\right)$ was calculated from the slope of the linear relationship between the $\log _{\mathrm{e}}$ concentration and the time during the terminal elimination phase. AUC from time zero to the time of the last measurable plasma concentration $\left(\mathrm{AUC}_{t}\right)$ was calculated by the linear trapezoidal rule, and AUC from time zero to infinity $\left(\mathrm{AUC}_{\infty}\right)$ was calculated after extrapolation from time $t$ to infinity as the ratio of the last measurable plasma concentration $\left(C_{\text {last }}\right)$ to $k_{\mathrm{e}}$. The elimination half-life $\left(t_{1 / 2}\right)$ was estimated from the elimination rate constant as $\ln (2) / k_{\mathrm{e}}$. 


\subsection{Statistical Methods}

Statistical analysis was performed using the validated program SPSS v22.0. The ratios used to test bioequivalence were calculated from $\log _{\mathrm{e}}$-transformed data for $C_{\max }, \mathrm{AUC}_{t}$ and $\mathrm{AUC}_{\infty}$. The differences between the $\log _{\mathrm{e}}$ means and the $90 \%$ confidence intervals (CIs) of the differences, derived from the residual variance from the analysis of variance (ANOVA) model, were back-transformed to estimate the ratios of the two formulations and the $90 \%$ CIs of these ratios.

\subsection{Safety}

Safety was assessed in terms of the overall proportion of subjects with adverse events (AEs) and by haematological and biochemical assessment of blood samples. AEs were evaluated for their severity (mild, moderate or severe) according to the subjective impact they had on the performance of daily activities and their likely relationship to the medication (not related, unlikely, possibly, probably or definitely related) according to the likelihood of a temporal association between the onset of the event and the administration of the medicinal product.

\section{Results}

\subsection{Participants}

Thirty subjects were enrolled in this study; 23 (77\%) were male, and all were Caucasian. The mean ( \pm standard deviation) age and BMI were $29.9( \pm 19.4)$ years and 24.5 $( \pm 2.9) \mathrm{kg} / \mathrm{m}^{2}$, respectively. Twenty-nine participants completed the study, each of whom received all five treatments and was included in the pharmacokinetic analysis.

\subsection{Pharmacokinetic Results}

The mean paracetamol plasma concentration-time curves for ibuprofen and paracetamol are presented in Figs. 1 and 2, respectively. A tabulated summary of the pharmacokinetic data for both compounds is also presented in Table 2 .

Intravenous infusions resulted in mean $C_{\max }$ values of 26,709.6 ng/mL (FDC-IV) and 26,236 ng/mL (paracetamol IV) immediately after the 15-min infusion. A half dose of FDC-IV provided a mean $C_{\max }$ of $12,880 \mathrm{ng} / \mathrm{mL}$. The concentration of paracetamol from the FDC-oral tablets peaked at $0.73 \mathrm{~h}$, with a mean $C_{\max }$ of $14,907 \mathrm{ng} / \mathrm{mL}$. The oral route provided paracetamol $C_{\max }$ values $44 \%$ lower than those observed after administration of FDC-IV or paracetamol IV.

Similarly, IV infusions resulted in $C_{\max }$ values of $39,506.7 \mathrm{ng} / \mathrm{mL}$ (FDC-IV) and $40,292.97 \mathrm{ng} / \mathrm{mL}$

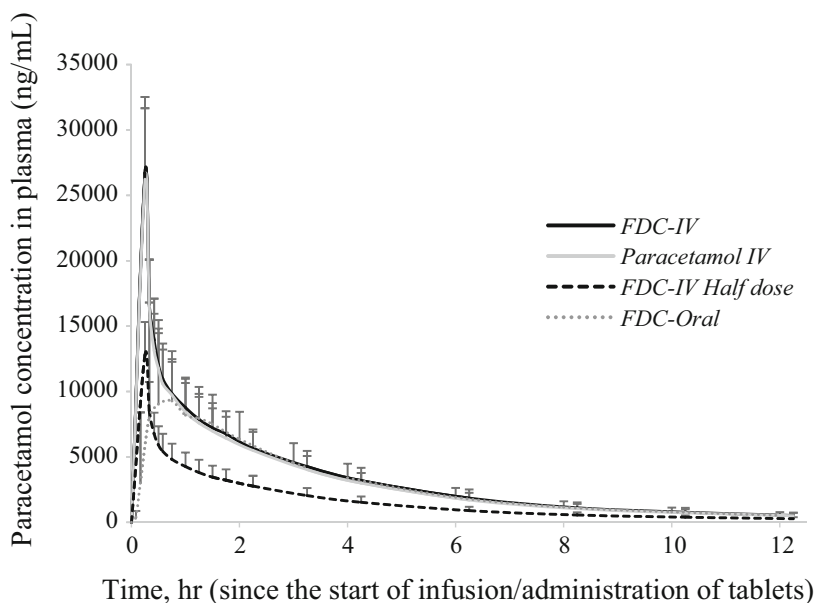

Fig. 1 Mean (standard error of the mean) paracetamol plasma concentrations after single administration of FDC-IV (treatment A: $3 \mathrm{mg} / \mathrm{mL}$ ibuprofen $+10 \mathrm{mg} / \mathrm{mL}$ paracetamol, $100 \mathrm{~mL} \mathrm{IV),} \mathrm{parac-}$ etamol IV (treatment B: $10 \mathrm{mg} / \mathrm{mL}$ paracetamol, $100 \mathrm{~mL}$ IV), FDCIV half dose (treatment D: $1.5 \mathrm{mg} / \mathrm{mL}$ ibuprofen $+5 \mathrm{mg} / \mathrm{mL}$ paracetamol, $100 \mathrm{~mL} \mathrm{IV)} \mathrm{and} \mathrm{FDC-oral} \mathrm{(treatment} \mathrm{E:} \mathrm{ibuprofen}$ $300 \mathrm{mg}+$ paracetamol $1000 \mathrm{mg}, 2$ tablets). $F D C$ fixed-dose combination, $I V$ intravenous

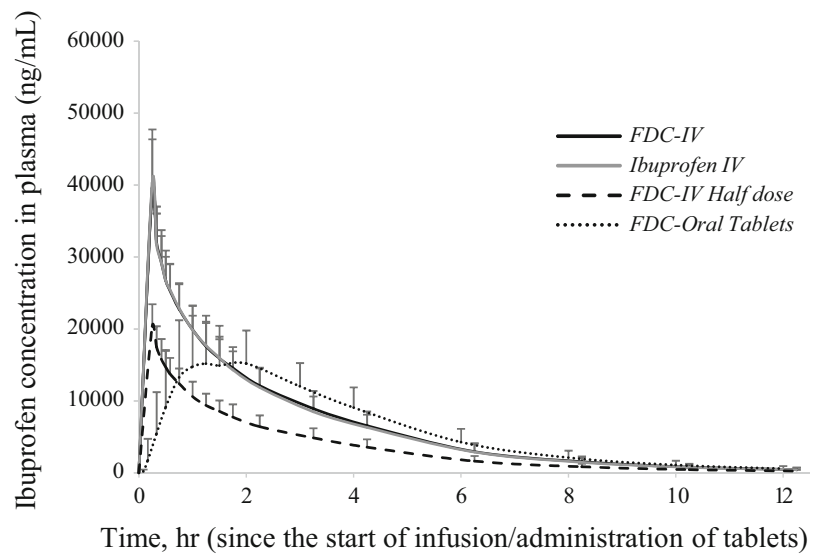

Fig. 2 Mean (standard error of the mean) ibuprofen plasma concentrations after single administration of FDC-IV (treatment A: $3 \mathrm{mg} / \mathrm{mL}$ ibuprofen $+10 \mathrm{mg} / \mathrm{mL}$ paracetamol, $100 \mathrm{~mL} \mathrm{IV),} \mathrm{ibuprofen} \mathrm{IV}$ (treatment C: $3 \mathrm{mg} / \mathrm{mL}$ ibuprofen, $100 \mathrm{~mL} \mathrm{IV),} \mathrm{FDC-IV} \mathrm{half} \mathrm{dose}$ (treatment D: $1.5 \mathrm{mg} / \mathrm{mL}$ ibuprofen $+5 \mathrm{mg} / \mathrm{mL}$ paracetamol, $100 \mathrm{~mL} \mathrm{IV)} \mathrm{and} \mathrm{FDC-oral} \mathrm{(treatment} \mathrm{E:} \mathrm{ibuprofen} 300 \mathrm{mg}+$ paracetamol $1000 \mathrm{mg}, 2$ tablets). $F D C$ fixed-dose combination, $I V$ intravenous

(ibuprofen IV) immediately after the 15 -min infusion. A half dose of FDC-IV provided a mean $C_{\max }$ of $20,352 \mathrm{ng} /$ $\mathrm{mL}$. The concentration of ibuprofen from the FDC-oral tablets peaked at $1.49 \mathrm{~h}$, with a mean $C_{\max }$ of $19,637 \mathrm{ng} /$ $\mathrm{mL}$. The oral route provided ibuprofen $C_{\max }$ values $50 \%$ lower than those observed after administration of FDC-IV or ibuprofen IV.

The corresponding $\mathrm{AUC}_{t}$ and $\mathrm{AUC}_{\infty}$ values were very similar across both the full-dose IV formulation and the 
FDC-oral tablets. The back-transformed $90 \% \mathrm{CIs}$ for FDC-IV in comparison with paracetamol IV and ibuprofen IV all fell within the 80-125\% acceptable bioequivalence range, thus confirming the lack of any pharmacokinetic interaction when both paracetamol and ibuprofen are given in combination as a parenteral preparation (Table 3).

Comparisons of the $C_{\max }, \mathrm{AUC}_{t}$ and $\mathrm{AUC}_{\infty}$ values for FDC-IV and a half dose of FDC-IV demonstrated consistent dose proportionality for both paracetamol and ibuprofen (Table 4). The $t_{1 / 2}$ values for paracetamol and ibuprofen were found to be comparable between all treatment groups (Table 2).

The relative bioavailability of paracetamol and ibuprofen from the FDC-oral tablets was calculated from the back-transformed ratios of the $\log _{\mathrm{e}}$ means of the $\mathrm{AUC}_{\infty}$ values for the oral and IV formulations ( $\mathrm{AUC}_{\mathrm{FDC} \text {-oral }}$ AUC $\left._{\text {FDC-IV }}\right)$. For FDC-oral, the relative bioavailability of paracetamol was $93.78 \%$ (90\% CI 90.98-96.67\%) and the absolute bioavailability of ibuprofen was $96.45 \%$ (90\% CI 93.13-99.89\%), suggesting nearly complete absorption of both compounds from the gastrointestinal mucosa. These results are summarized in Table 5.

\subsection{Safety Analysis}

Twenty-five non-serious AEs were reported by 15 subjects (50\%). The majority of AEs were mild (80\%), and all other AEs were moderate $(20 \%)$. There were no severe AEs. Seventy-six percent of AEs were not related to the study medication in that a temporal association between the onsets of the events relative to the administration of the product was not reasonable. Twenty-four percent of AEs had a reasonable temporal relationship between their onset and administration of the study medication, and were deemed either 'unlikely related' or 'possibly related' on the basis of the subjective likelihood of the association. These data, and the distribution of AEs across the study periods (study drug or washout), are presented in Table 6. One subject did suffer a serious AE: bilateral ankle fractures due to a fall.

Table 2 Mean \pm standard deviation pharmacokinetic parameters of paracetamol and ibuprofen from each treatment

\begin{tabular}{|c|c|c|c|c|}
\hline \multirow[t]{2}{*}{ Paracetamol } & \multicolumn{4}{|c|}{ Treatment (mean \pm standard deviation) } \\
\hline & FDC-IV: treatment A & Paracetamol IV: treatment B & FDC-IV half dose: treatment D & FDC-oral: treatment $\mathrm{E}$ \\
\hline$C_{\max }(\mathrm{ng} / \mathrm{mL})$ & $26,709.57 \pm 5814.74$ & $26,236.06 \pm 5430.52$ & $12,880.39 \pm 2553.15$ & $14,907.16 \pm 6255.10$ \\
\hline $\mathrm{AUC}_{t}(\mathrm{ng} \cdot \mathrm{h} / \mathrm{mL})$ & $37,553.97 \pm 9816.96$ & $35,846.20 \pm 8734.15$ & $18,327.40 \pm 4758.34$ & $34,980.80 \pm 9430.21$ \\
\hline $\mathrm{AUC}_{\infty}(\mathrm{ng} \cdot \mathrm{h} / \mathrm{mL})$ & $39,419.95 \pm 10,630.63$ & $37,651.43 \pm 9454.60$ & $19,337.01 \pm 5146.46$ & $37,023.82 \pm 10,388.31$ \\
\hline$T_{\max }(\mathrm{h})^{\mathrm{a}}$ & $0.00 \pm 0.00$ & $0.00 \pm 0.00$ & $0.00 \pm 0.02$ & $0.73 \pm 0.42$ \\
\hline$t_{1 / 2}(\mathrm{~h})$ & $2.39 \pm 0.27$ & $2.38 \pm 0.25$ & $2.44 \pm 0.25$ & $2.51 \pm 0.33$ \\
\hline \multirow[t]{2}{*}{ Ibuprofen } & \multicolumn{4}{|c|}{ Treatment (mean \pm standard deviation) } \\
\hline & FDC-IV: treatment A & Ibuprofen IV: treatment $\mathrm{C}$ & FDC-IV half dose: treatment D & FDC-oral: treatment E \\
\hline$C_{\max }(\mathrm{ng} / \mathrm{mL})$ & $39,506.69 \pm 6874.06$ & $40,292.97 \pm 7460.04$ & $20,352.05 \pm 3090.87$ & $19,637.38 \pm 5178.29$ \\
\hline $\mathrm{AUC}_{t}(\mathrm{ng} \cdot \mathrm{h} / \mathrm{mL})$ & $73,492.69 \pm 16,509.61$ & $72,169.59 \pm 15,608.70$ & $39,642.48 \pm 9679.16$ & $70,417.75 \pm 16,260.16$ \\
\hline $\mathrm{AUC}_{\infty}(\mathrm{ng} \cdot \mathrm{h} / \mathrm{mL})$ & $74,743.31 \pm 17,388.69$ & $73,410 \pm 16,500.76$ & $40,333.88 \pm 10,240.30$ & $72,202.48 \pm 17,445.46$ \\
\hline$T_{\max }(\mathrm{h})^{\mathrm{a}}$ & $0.00 \pm 0.00$ & $0.00 \pm 0.00$ & $0.00 \pm 0.00$ & $1.49 \pm 0.89$ \\
\hline$t_{1 / 2}(\mathrm{~h})$ & $1.87 \pm 0.27$ & $1.88 \pm 0.28$ & $1.88 \pm 0.30$ & $1.99 \pm 0.36$ \\
\hline
\end{tabular}

$A U C_{t}$ from time zero to the time of the last measurable plasma concentration, $A U C_{\infty}$ area under the plasma concentration-time curve from time zero to infinity, $C_{\max }$ maximum measured plasma concentration, $t_{1 / 2}$ half-life, $T_{\max }$ time to reach $C_{\max }$

${ }^{\text {a }}$ Hours after the end of administration

Table 3 Bioequivalence comparison of FDC-IV (treatment A) and standalone IV preparations of paracetamol (treatment B) and ibuprofen (treatment C) $C_{\max }, \mathrm{AUC}_{t}$ and $\mathrm{AUC}_{\infty}$ [point estimate $\%(90 \% \mathrm{CI})$ from log-transformed data]

\begin{tabular}{llr}
\hline & Paracetamol: treatment A/B & Ibuprofen: treatment A/C \\
\hline$C_{\max }(\mathrm{ng} / \mathrm{mL})$ & $101.67(95.97-107.72)^{\mathrm{a}}$ & $98.30(93.82-102.99)^{\mathrm{a}}$ \\
$\mathrm{AUC}_{t}(\mathrm{ng} \cdot \mathrm{h} / \mathrm{mL})$ & $104.24(101.42-107.13)^{\mathrm{a}}$ & $101.57(98.35-104.89)^{\mathrm{a}}$ \\
$\mathrm{AUC}_{\infty}(\mathrm{ng} \cdot \mathrm{h} / \mathrm{mL})$ & $104.15(101.13-107.26)^{\mathrm{a}}$ & $101.55(93.82-105.00)^{\mathrm{a}}$ \\
\hline
\end{tabular}

$A U C_{t}$ from time zero to the time of the last measurable plasma concentration, $A U C_{\infty}$ area under the plasma concentration-time curve from time zero to infinity, $C I$ confidence interval, $C_{\max }$ maximum measured plasma concentration, $F D C$ fixed-dose combination, $I V$ intravenous

${ }^{a}$ Within the bioequivalence range 
Table 4 Dose proportionality of FDC-IV, expressed as a comparison between FDC-IV (treatment A) and FDC-IV half dose (treatment D) for paracetamol and ibuprofen $C_{\max }, \mathrm{AUC}_{t}$ and $\mathrm{AUC}_{\infty}$ [point estimate \% $(90 \% \mathrm{CI})$ from log-transformed data]

\begin{tabular}{llr}
\hline & Paracetamol: treatment A/D & Ibuprofen: treatment A/D \\
\hline$C_{\max }(\mathrm{ng} / \mathrm{mL})$ & $206.89(196.27-218.08)$ & $193.49(185.70-201.61)$ \\
$\mathrm{AUC}_{t}(\mathrm{ng} \cdot \mathrm{h} / \mathrm{mL})$ & $204.49(199.31-209.79)$ & $185.84(181.10-190.71)$ \\
$\mathrm{AUC}_{\infty}(\mathrm{ng} \cdot \mathrm{h} / \mathrm{mL})$ & $203.89(198.37-208.58)$ & $185.81(180.90-190.87)$ \\
\hline
\end{tabular}

$A U C_{t}$ from time zero to the time of the last measurable plasma concentration, $A U C_{\infty}$ area under the plasma concentration-time curve from time zero to infinity, $C I$ confidence interval, $C_{\max }$ maximum measured plasma concentration, $F D C$ fixed-dose combination, $I V$ intravenous

Table 5 Relative bioavailability of FDC-oral determined by ratios of FDC-oral and FDC-IV geometric means of ibuprofen and paracetamol $\mathrm{AUC}_{\infty}$ values [point estimate $\%(90 \% \mathrm{CI})$ from log-transformed data]

\begin{tabular}{|c|c|c|c|}
\hline & FDC-oral: treatment $\mathrm{E}^{\mathrm{a}}$ & FDC-IV: treatment $\mathrm{A}^{\mathrm{a}}$ & Relative bioavailability: treatment E/A \\
\hline Paracetamol AUC $\mathrm{AO}_{\infty}(\mathrm{ng} \cdot \mathrm{h} / \mathrm{mL})$ & $35,721.16$ & $38,091.23$ & $93.78(90.98-96.67)^{\mathrm{b}}$ \\
\hline Ibuprofen $\mathrm{AUC}_{\infty}(\mathrm{ng} \cdot \mathrm{h} / \mathrm{mL})$ & $70,233.598$ & $72,814.966$ & $96.45(93.13-99.89)^{\mathrm{b}}$ \\
\hline
\end{tabular}

$A U C_{\infty}$ area under the plasma concentration-time from time zero to infinity, $C I$ confidence interval, $F D C$ fixed-dose combination, $I V$ intravenous

a Geometric mean

b Within the bioequivalence range

\section{Discussion}

For oral medications, increased absorption time and firstpass metabolism, or inconsistent absorption in hospitalized patients, due to delayed gastric emptying perioperatively, may result in a latency to or lack of clinical effect [15]. Indeed, in a study of 106 patients undergoing ear, nose and throat surgery, plasma concentrations of paracetamol $1000 \mathrm{mg}$ sufficient to provide an analgesic effect $(10 \mathrm{mg} / \mathrm{L})$ were reached in $96 \%$ of patients who received the drug parenterally and in only $67 \%$ patients who received it orally [16]. FDC-IV has been developed for use in instances where oral therapy is limited by sedation, severe illness or debilitation, or in patients with difficulty in swallowing.

\subsection{Interaction}

The results of this study demonstrate that concurrent administration of ibuprofen and paracetamol in a novel IV fixed-dose combination did not significantly alter the extent of absorption of ibuprofen or paracetamol, in comparison with either agent alone. A lack of a pharmacokinetic interaction between ibuprofen and paracetamol has been documented for the following oral formulation, fixed-dose combinations: ibuprofen $300 \mathrm{mg}+$ paracetamol $1000 \mathrm{mg}$ [10], ibuprofen $400 \mathrm{mg}+$ paracetamol $1000 \mathrm{mg}$ [11] and ibuprofen $400 \mathrm{mg}+$ paracetamol $650 \mathrm{mg}$ [12]. In these products, after oral administration, the values of the pharmacokinetic parameters $C_{\max }, \mathrm{AUC}_{t}$ and $\mathrm{AUC}_{\infty}$ for both ibuprofen and paracetamol were similar with the fixed-dose combinations and monotherapies, and the back-transformed
$90 \%$ CI for each parameter fell within the acceptable bioequivalence range $(80-125 \%)[10,11]$. Similar results were obtained from comparison of pharmacokinetic parameters by ANOVA for the third fixed-dose combination [12]. Consequently, the results from the present study confirm and extend such results for an IV formulation.

\subsection{Dose Proportionality}

The dose proportionality of the FDC-IV formulation provided here is consistent with that reported for IV ibuprofen; a twofold lesser dose results in decreases of $44-51 \%$ in $C_{\max }, \mathrm{AUC}_{t}$ and $\mathrm{AUC}_{\infty}[6,14]$. Absorption of paracetamol from a half dose of FDC-IV appeared to be slightly delayed; however, the longer mean $T_{\max }$ in this group was due to a single patient whose paracetamol $C_{\max }$ occurred 20 min after the start of the 15-min infusion (5 min after the end of the infusion). The clearance of both compounds remained constant $(2.4 \mathrm{~h}$ for paracetamol and $1.8 \mathrm{~h}$ for ibuprofen), which is consistent with previous findings for ibuprofen [6]. The clear dose proportionality of absorption of both active ingredients in FDC-IV could provide practitioners with the ability to easily titrate analgesia according to their patient's pain.

\subsection{Relative Bioavailability}

This study determined that both active ingredients in the FDC-oral formulation have very high bioavailability. In comparison with FDC-IV, the relative bioavailability values for ibuprofen and paracetamol in the FDC-oral tablets 
Table 6 Distribution of adverse events by study period/treatment, severity and relationship with medication

\begin{tabular}{|c|c|c|}
\hline \multirow[t]{2}{*}{ Treatment period and adverse event } & \multicolumn{2}{|c|}{ Grading of adverse events } \\
\hline & Severity $^{\mathrm{a}}$ & Relationship to study medication ${ }^{b}$ \\
\hline \multicolumn{3}{|l|}{ FDC-IV } \\
\hline Right superficial radial nerve irritation secondary to cannulation on right wrist & Mild & Not related \\
\hline Bruised cannulation site on left forearm & Mild & Not related \\
\hline Upper respiratory tract infection & Mild & Not related \\
\hline Nausea & Mild & Possibly related \\
\hline \multicolumn{3}{|l|}{ Paracetamol IV } \\
\hline Upper respiratory tract infection & Mild & Not related \\
\hline Lethargy & Mild & Possibly related \\
\hline Vasovagal episode & Moderate & Not related \\
\hline Headache & Moderate & Not related \\
\hline Dyspepsia & Moderate & Possibly related \\
\hline \multicolumn{3}{|l|}{ Ibuprofen IV } \\
\hline Upper respiratory tract infection & Mild & Not related \\
\hline Rectal bleeding & Mild & Unlikely related \\
\hline Diarrhoea & Moderate & Unlikely related \\
\hline \multicolumn{3}{|l|}{ FDC-IV half dose } \\
\hline Sore throat & Mild & Not related \\
\hline \multicolumn{3}{|l|}{ FDC-oral } \\
\hline Vasovagal episode & Mild & Not related \\
\hline Bruising at cannula site on left forearm & Mild & Not related \\
\hline Presyncopal episode & Moderate & Not related \\
\hline \multicolumn{3}{|l|}{ Washout } \\
\hline Upper respiratory tract infection & Mild & Not related \\
\hline Dry cough & Mild & Not related \\
\hline Nasal congestion & Mild & Not related \\
\hline Headache & Mild & Not related \\
\hline Headache & Mild & Not related \\
\hline Upper respiratory tract infection & Mild & Not related \\
\hline Bruising at cannula site on right forearm & Mild & Not related \\
\hline Bruising at failed cannula site on right wrist & Mild & Not related \\
\hline Rectal bleeding & Mild & Unlikely related \\
\hline
\end{tabular}

FDC fixed-dose combination, $I V$ intravenous

a Mild: discomfort noticed but no disruption of normal daily activity; moderate: discomfort sufficient to reduce or affect daily activity

b Unlikely related: a temporal (timely) relationship of the onset of the event, relative to the administration of the product, is unlikely but cannot be ruled out; possibly related: a temporal (timely) relationship of the onset of the event, relative to the administration of the product, is reasonable, but the event could have been due to an equally likely cause

are 93.59 and $96.34 \%$ respectively, confirming the assertion that both compounds are not subject to significant presystemic metabolism. These results are congruent with previously published data [5-9].

Absorption via the gastrointestinal mucosa results in delayed absorption and decreased $C_{\max }$ values. In comparison with FDC-IV, the $C_{\max }$ values for ibuprofen and paracetamol were reduced by 50 and $44 \%$, respectively, after administration of FDC-oral. For ibuprofen, the results of this study are in line with other published data; an equivalent oral dose of ibuprofen results in a $C_{\max }$ value that is $50 \%$ of the value observed after an IV dose (63 versus $120 \mu \mathrm{g} / \mathrm{mL}$ ) [5]. For paracetamol, oral dosing has been shown to reduce $C_{\max }$ of paracetamol $1000 \mathrm{mg}$ by $41-43 \%$ in healthy volunteers $[9,13]$.

\section{Conclusion}

This study confirmed that a combination of $3 \mathrm{mg} / \mathrm{mL}$ ibuprofen and $10 \mathrm{mg} / \mathrm{mL}$ paracetamol (FDC-IV) produces ibuprofen and paracetamol plasma concentration-time 
profiles that are similar to those observed after IV administration of each component on its own. The pharmacokinetic profile of the IV formulation has been demonstrated to be dose proportional following administration of single doses of $1.5 \mathrm{mg} / \mathrm{mL}$ ibuprofen and $5 \mathrm{mg} / \mathrm{mL}$ paracetamol (FDC-IV half dose) and $3 \mathrm{mg} / \mathrm{mL}$ ibuprofen and $10 \mathrm{mg} /$ $\mathrm{mL}$ paracetamol (FDC-IV).

The pharmacokinetic parameters were observed to be similar when a single dose of FDC 300/1000 was administered in either an IV formulation (FDC-IV) or an oral formulation (FDC-oral), except for $C_{\max }$ of the IV formulation, which was twice that of the oral formulation, and, as expected, $T_{\max }$ was much shorter than with the oral dose. The relative bioavailability of paracetamol $(93.78 \%)$ and ibuprofen $(96.45 \%)$ confirmed the pharmacokinetic equivalence of the oral and IV FDC 300/1000 formulations.

Consequently, in instances where delayed gastric emptying is expected, an IV formulation of FDC 300/1000 may provide more efficient analgesic delivery.

Acknowledgments We thank the staff at Christchurch Clinical Studies Trust Ltd (CCST; Christchurch, New Zealand) for the administration of the study protocol and data collection. We thank the staff at the International Pharmaceutical Research Centre (IPRC; Amman, Jordan) for the data analysis. We are grateful to the volunteers who participated in this study. This study was funded by AFT Pharmaceuticals Ltd (Auckland, New Zealand).

Author Contributions H. Atkinson supervised the project and was involved in the design of the study, development of the protocol, interpretation of the data and writing of the manuscript. I. Stanescu was involved in the study design, protocol development, data analysis, data interpretation and writing of the manuscript. C. Frampton conducted the statistical analysis of the data and reviewed the manuscript. I. Salem performed the plasma drug assays and reviewed the manuscript. C. Beasley contributed to the statistical data interpretation and assisted in drafting the manuscript. R. Robson was involved in the data interpretation and critically reviewed the manuscript. All authors had full access to all of the data.

The authors have full control of all primary data and agree to allow the journal to review their data if requested.

\section{Compliance with Ethical Standards}

The study protocol received approval by the Health and Disability Ethics Committee, Ministry of Health, New Zealand, and was conducted in accordance with Good Clinical Practice (GCP), including the Declaration of Helsinki. Informed consent was obtained from all individual participants prior to their inclusion in the study.

This study was funded by AFT Pharmaceuticals Ltd.

H. Atkinson is a shareholder and Managing Director of AFT Pharmaceuticals Ltd, and I. Stanescu and C. Beasley are employees of AFT Pharmaceuticals Ltd. I. Salem is an employee of the IPRC. C. Frampton provides consultancy services to AFT Pharmaceuticals Ltd. R. Robson is the Director of CCST.

Open Access This article is distributed under the terms of the Creative Commons Attribution-NonCommercial 4.0 International License (http://creativecommons.org/licenses/by-nc/4.0/), which permits any noncommercial use, distribution, and reproduction in any medium, provided you give appropriate credit to the original author(s) and the source, provide a link to the Creative Commons license, and indicate if changes were made.

\section{References}

1. Bailey E, Worthington HV, van Wijk A, Yates JM, Coulthard P, Afzal Z. Ibuprofen and/or paracetamol (acetaminophen) for pain relief after surgical removal of lower wisdom teeth. Cochrane Database Syst Rev. 2013;12:CD004624. doi:10.1002/14651858. CD004624.pub2.

2. Malmberg AB, Yaksh TL. Cyclooxygenase inhibition and the spinal release of prostglandin E2 and amino acids evoked by paw formalin injection: a microdialysis study in unanesthetized rats. J Neurosci. 1995; 15:2768-78.

3. Anderson BJ. Paracetamol (acetaminophen): mechanisms of action. Paediatr Anaesth. 2008;18:915-21.

4. Merry AF, Gibbs RD, Edwards J, Ting GS, Frampton C, Davies E, Anderson BJ. Combined acetaminophen and ibuprofen for pain relief after oral surgery in adults: a randomized controlled trial. Br J Anaesth. 2010;104:80-8.

5. Pavliv L, Voss B, Rock A. Pharmacokinetics, safety, and tolerability of a rapid infusion of i.v. ibuprofen in healthy adults. Am J Health Syst Pharm. 2011;68:47-51.

6. Martin W, Koselowske G, Töberich H, Kerkmann T, Mangold B, Augustin J. Pharmacokinetics and absolute bioavailability of ibuprofen after oral administration of ibuprofen lysine in man. Biopharm Drug Dispos. 1990;11:265-78.

7. Perucca E, Richens A. Paracetamol disposition in normal subjects and in patients treated with antiepileptic drugs. Br J Clin Pharmacol. 1979;7:201-6.

8. Rawlins MD, Henderson DB, Hijab AR. Pharmacokinetics of paracetamol (acetaminophen) after intravenous and oral administration. Eur J Clin Pharmacol. 1977;11:283-6.

9. Singla NK, Parulan C, Samson R, Hutchinson J, Bushnell R, Beja $\mathrm{EG}$, et al. Plasma and cerebrospinal fluid pharmacokinetic parameters after single-dose administration of intravenous, oral, or rectal acetaminophen. Pain Pract. 2012;12:523-32.

10. Atkinson HC, Stanescu I, Beasley CPH, Salem II, Frampton C. A pharmacokinetic analysis of a novel fixed dose oral combination of paracetamol and ibuprofen, with emphasis on food effect. J Bioequiv Availab. 2015;7:150-4.

11. Tanner T, Aspley S, Munn A, Thomas T. The pharmacokinetic profile of a novel fixed-dose combination tablet of ibuprofen and paracetamol. BMC Pharmacol Toxicol. 2010;10:10.

12. Wright CE, Antal EJ, Gillespie WR, Albert KS. Ibuprofen and acetaminophen kinetics when taken concurrently. Clin Pharmacol Ther. 1983;34:707-10.

13. Cadence Pharmaceuticals, Inc. Product monograph: Ofirmev (acetaminophen) injection. 2010. http://c.ymcdn.com/sites/www. npamonline.org/resource/resmgr/imported/OFIRMEV\% 20Monograph_FINAL.pdf. Accessed 12 Jan 2015.

14. Cumberland Pharmaceuticals. Product monograph: Caldolor (ibuprofen for intravenous injection). Nashville: Cumberland Pharmaceuticals Inc; 2009.

15. Power DBM, Forbes AM, van Heerden PV, Ilett APKF. Pharmacokinetics of drugs used in critically ill adults. Clin Pharmacokinet. 1998;34:25-56.

16. Van Der Westhuizen J, Kuo PY, Reed EW, Holder K. Randomised controlled trial comparing oral and intravenous paracetamol (acetaminophen) plasma levels when given as preoperative analgesia. Anaesth Intensiv Care. 2011;39:242-6. 